\title{
RISCO CARDIOVASCULAR DE USUÁRIOS ATIVOS, INSUFICIENTEMENTE ATIVOS E INATIVOS DE PARQUES PÚBLICOS
}

\author{
CARDIOVASCULAR RISK IN ACTIVE, INSUFFICIENTLY ACTIVE AND INACTIVE \\ USERS OF PUBLIC PARKS
}

\begin{abstract}
RESUMO
Introdução: A prática de atividades físicas tem sido recomendada para a prevenção e reabilitação cardíacas. Porém, quando feita de maneira inadequada, o que ocorre mais freqüentemente na ausência de supervisão em locais públicos, esta prática pode se associar ao aumento do risco de acometimentos cardiovasculares. Objetivo: Comparar o risco cardiovascular de freqüentadores de parques públicos de São Paulo com diferentes níveis de prática de atividade física - ativos, insuficientemente ativos e inativos. Métodos: A avaliação constou de questionário sobre a presença de doenças, sintomas e fatores de risco cardiovasculares e sobre a prática de atividade física, além de medidas antropométricas e da pressão arterial. Resultados: Não houve diferença significante na prevalência de doenças e fatores de risco controláveis entre os grupos. Porém, os inativos apresentaram maior prevalência de sintomas cardiovasculares (34\%). Quanto aos fatores não-controláveis, a prevalência do fator sexo/idade foi maior nos ativos (50\%) e insuficientemente ativo (45\%), e a hereditariedade foi mais observada nos inativos (35\%). Além disso, não foram observadas diferenças entre os grupos nas medidas de obesidade e pressão arterial. Por fim, observou-se que os ativos e insuficientemente ativos têm maior conhecimento sobre sua saúde e possuem mais recomendação médica para a prática de atividade física. Conclusão: Esses dados indicam que grande parte dos praticantes de atividades físicas nos parques é formada por idosos e apresenta risco cardiovascular moderado a alto para esta prática, o que levanta a importância da presença de um profissional da área de atividade física nesses locais.
\end{abstract}

Palavras-chave: Doenças cardiovasculares; Fatores de risco; Atividade motora; Áreas verdes.

\section{ABSTRACT}

Physical activity has been recommended for heart disease prevention and rehabilitation. However, when performed incorrectly, which is more common when practiced without supervision and in public places, the risk of cardiovascular events increases. The objective of this study was to compare cardiovascular risk factors among users of São Paulo's public parks with differing levels of physical activity - active, insufficiently active, and inactive. The evaluation consisted of a questionnaire about cardiovascular diseases, symptoms and risk factors; physical activity practice; and anthropometric and arterial blood pressure measurements. There was no difference between the groups in terms of the prevalence of cardiovascular disease or controllable risk factors. However, inactive people had a higher prevalence of cardiovascular symptoms (35\%). With regard to uncontrollable cardiovascular risk factors, there was a higher prevalence of the gender/age factor among active $(50 \%)$ and insufficiently active (45\%) subjects, and heredity was more prevalent among inactive people (35\%). There was no difference in obesity or blood pressure between the groups. The study also showed that active and insufficiently active subjects have a better knowledge of their health status, and a higher prevalence of being prescribed physical activity by physicians. The results demonstrate that most of the people who exercise in public parks are elderly and are at a moderate to high cardiovascular risk from this practice, which suggests that a physical education professional should be present.

Key words: Cardiovascular diseases; Risk factors; Motor activity; Green areas.

1 Laboratório de Hemodinâmica da Atividade Motora da Escola de Educação Física e Esporte da Universidade de São Paulo. Brasil 


\section{INTRODUÇÃO}

As doenças cardiovasculares são responsáveis por aproximadamente $33 \%$ do total de mortes no Brasil ${ }^{1}$. A alta prevalência destas doenças e de seus fatores de risco (hipertensão, tabagismo, diabetes, dislipidemia, obesidade e sedentarismo) tem atentado as autoridades para a necessidade de controle dos mesmos.

Entre os fatores de risco para as doenças cardiovasculares, o sedentarismo parece ser muito importante devido a sua alta prevalência e sua influência sobre os outros fatores. De fato, estudos ${ }^{2,3}$ mostram uma relação inversa entre a prática regular de atividades físicas e a incidência de problemas cardíacos. Indivíduos que têm gasto energético semanal de 1000 a $2500 \mathrm{kcal}$ em atividades de lazer, possuem 20\% menos risco de desenvolver doença da artéria coronária que indivíduos inativos ${ }^{4}$. Além disso, dentre os ativos, aqueles que se exercitam mais têm menor chance de desenvolver doenças do coração ${ }^{2}$. Assim, a prática regular de atividades físicas tem sido recomendada para a prevenção e reabilitação cardíacas ${ }^{5,6}$.

A atividade física apresenta o impacto benéfico exposto anteriormente devido a seus efeitos sobre o sistema cardiovascular e sobre os fatores de risco. Neste sentido, o condicionamento físico adequado fortalece o músculo do coração e modifica a estrutura vascular coronariana, auxiliando no tratamento de doenças ligadas ao sistema circulatório ${ }^{2,3,5,7}$. Além disso, ele reduz a pressão arterial sistólica (PAS) e diastólica (PAD) em 3,3 e 3,5 mmHg, respectivamente ${ }^{8,9}$, diminui a concentração de hemoglobina glicada em $66 \%$, mostrando sua eficiência no controle glicêmico e na prevenção do diabetes melitus ${ }^{10}$. O perfil lipídico também pode se modificar com a prática de atividade física, de modo que se observam reduções nas concentrações de triglicérides, LDL-colesterol e colesterol total de 4,5 e $1 \%$, respectivamente, além de aumento de $5 \%$ do HDL-colesterol ${ }^{11}$. Além disso, a prática de atividades físicas está relacionada à redução do peso corporal, do índice de massa corporal e do índice cintura-quadril ${ }^{11}$.

Mesmo com os benefícios comprovados da prática de atividades físicas para o sistema cardiovascular, a prevalência do sedentarismo em nosso país é bastante elevada. No município de São Paulo, 69\% da população adulta é sedentária ${ }^{12}$. Um estudo mais recente ${ }^{13}$ mostra que $87 \%$ dos brasileiros não praticam nenhuma atividade física de lazer regularmente. Assim, diversos programas ${ }^{14}$ têm surgido no intuito de tornar a população mais ativa. Estes têm levado as pessoas a realizar suas atividades em diferentes locais e, muitas vezes em locais públicos, como ruas, praças e parques. Nos Estados Unidos, dentre as pessoas que caminham, $60 \%$ o fazem nas ruas e, na região urbana, $35 \%$ utilizam os parques para tal ${ }^{4}$.

Apesar dos inúmeros benefícios e do incentivo ao aumento da prática de atividades físicas, é importante lembrar que durante a execução de atividades físicas há um aumento do trabalho cardíaco, o que pode gerar, em pessoas com problemas cardíacos conhecidos ou não, uma sobrecarga demasiada, levando a riscos imediatos de infarto do miocárdio ou parada cardíaca. De fato, o risco de infarto é seis vezes maior durante a prática de atividades físicas ${ }^{15}$. Além disso, esse risco é maior em indivíduos que possuem fatores de risco cardiovasculares e em cardiopatas, nos quais o risco é dez vezes maior que em indivíduos saudáveis ${ }^{5}$. É importante ressaltar que o risco é aumentado quando a prática é inadequada, intensa demais para o indivíduo ${ }^{15}$, e é mais freqüente quando a atividade é praticada sem orientação, o que pode ocorrer em locais públicos, que não possuem uma estrutura de orientação.

Diante do exposto, fica claro que as doenças cardiovasculares representam um grande problema de saúde pública e que a prática regular de atividade física pode ajudar na prevenção e reabilitação destas doenças. Assim, muitas pessoas têm realizado a prática de atividades físicas de lazer em parques públicos e, por vezes, sem orientação prévia. Por outro lado, a prática de atividade física inadequada, realizada principalmente por indivíduos cardiopatas e portadores de fatores de risco, pode se associar ao aumento de acometimentos cardiovasculares agudos durante a execução da atividade física. Desta forma, tornase importante observar qual o risco dos indivíduos que praticam atividades físicas em locais públicos, comparando-os aos indivíduos que freqüentam estes locais, mas não praticam atividades físicas. Como o nível de atividade parece ser um fator importante é interessante comparar os indivíduos com diferentes níveis de prática.

Assim, este estudo teve por objetivo descrever e comparar o risco cardiovascular de indivíduos ativos, insuficientemente ativos e inativos freqüentadores de parques municipais da região oeste do Município de São Paulo.

\section{PROCEDIMENTOS METODOLÓGICOS}

Foram avaliadas 749 freqüentadores de 7 parques públicos da zona oeste do município de São Paulo (Santa Felicidade, Fernando Costa, São Domingos, CEMUCAM, Alfredo Volpi, Luís Carlos Prestes e Previdência). A participação foi espontânea e aberta a todos os freqüentadores adultos dos parques. O estudo foi aprovado pelo Conselho de Ética da Escola de Educação Física e Esporte da Universidade de São Paulo (CEP10) e todos os voluntários assinaram o termo de consentimento.

Todos os voluntários responderam a um questionário aplicado na forma de entrevista que avaliou os dados pessoais, doenças, sintomas e fatores de risco cardiovascular e as atividades físicas de lazer praticadas. O questionário foi elaborado com base nas recomendações do American College of Sports Medicine e do American Heart Association ${ }^{5,16}$. Além disso, foram realizadas algumas medidas antropométricas e de pressão arterial.

A presença de doenças cardiovasculares foi considerada pelo relato direto do entrevistado de diagnóstico médico ou cirurgias/procedimentos 
que indicassem a presença de alguma doença cardiovascular ou, ainda, pelo uso de medicamentos específicos. A presença de sintomas cardiovasculares foi avaliada pelos relatos de dor no peito, falta de ar, tontura e desmaios, desde que estes sintomas fossem observados com freqüência e não fossem atribuídos a outras doenças. A presença dos fatores de risco - hipertensão, diabetes e hipercolesterolemia - foi avaliada através do relato direto ou do uso de medicamentos específicos para estes fatores.

Os outros fatores de risco cardiovasculares considerados foram: a) sexo/idade - se mulheres com mais de 55 anos ou homens com mais de 45 anos $^{5,16}$, b) hereditariedade - se pais, irmãos ou avós com histórico de doença cardiovascular antes dos 60 anos $^{1}$, c) fumo - pelo relato direto, d) sedentarismo - se o indivíduo não praticava atividades físicas de lazer regularmente, e e) obesidade - pelos indivíduos com índice de massa corporal (IMC) $\geq 30 \mathrm{~kg} / \mathrm{m}^{2}$.

O questionário avaliou também se os indivíduos possuíam alguma recomendação médica para a prática de atividades físicas e, se houvesse, qual o tipo de atividade recomendada.

Foram realizadas medidas de peso, estatura, perímetro da cintura e do quadril, e pressão arterial. $O$ peso foi medido em uma balança portátil com precisão de $1 \mathrm{~kg}$ e a estatura por um estadiômetro, graduado em centímetros. O cálculo do IMC foi feito através dequociente do peso $(\mathrm{kg})$ e da estatura ao quadrado $\left(\mathrm{m}^{2}\right)$. Os perímetros da cintura e do quadril foram medidos com uma fita métrica, sendo que a medida da cintura foi feita na altura do umbigo e a do quadril na maior circunferência. $O$ índice cintura/quadril (ICQ) foi calculado pelo quociente do valor do perímetro da cintura pelo quadril. Foram considerados altos os valores acima de 0,85 para mulheres e 1 para os homens. A pressão arterial foi medida uma vez pelo método auscultatório, após cinco minutos sentado em repouso. Nos indivíduos com circunferência de braço maior do que 33 centímetros, a medida foi corrigida pela fita de correção de pressão arterial ${ }^{17}$. Foram considerados altos os valores de PAS $\geq 140 \mathrm{mmHg}$ e/ou PAD $\geq 90 \mathrm{mmHg}^{6}$.

Os voluntários foram divididos em três grupos - ativos, insuficientemente ativos e inativos, de acordo com o volume semanal e a intensidade das atividades físicas de lazer praticadas ${ }^{18}$. Foram considerados ativos, os indivíduos que praticavam semanalmente no mínimo 150 min de atividades leves a moderadas ou mais do que 60 min de atividade intensa; insuficientemente ativos, os que realizavam atividades físicas com volume inferior ao citado; e inativos, os que não praticavam nenhuma atividade física regular. Assim, 406 indivíduos eram ativos, 137 insuficientemente ativos e 206 inativos.

Os dados são apresentados em valores percentuais e as comparações entre os grupos foram feitas utilizando-se o teste estatístico de qui-quadrado e considerando-se como significante o índice de $\mathrm{P}<0,05$.

\section{RESULTADOS}

Na população geral, havia 378 homens e 371 mulheres com uma idade média de 49+16 anos. Em relação ao risco cardiovascular, $13 \%$ da amostra apresentava doença cardíaca, 24\% tinha pelo menos um sintoma cardiovascular e $63 \%$ tinha pelo menos um fator de risco cardiovascular. De fato, $45 \%$ dos indivíduos apresentavam o fator sexo/idade, $28 \%$ hereditariedade, 27\% sedentarismo, 26\% hipertensão arterial, 24\% dislipidemia, $14 \%$ obesidade, $12 \%$ tabagismo e $7 \%$ diabetes melitus. Em relação à prática de atividade física de lazer, $73 \%$ praticavam regularmente, e destes, $65 \%$ faziam apenas atividades físicas aeróbias, 10\% praticavam regularmente atividades mistas, e $25 \%$ faziam ambas. Quanto às características dessa prática, $71 \%$ faziam pelo menos 150 minutos de atividade física semanais. Além disso, $23 \%$ o faziam em intensidade leve, $58 \%$ moderada e $19 \%$ intensa.

Como dito anteriormente, 406 indivíduos foram classificados como ativos, 137 como insuficientemente ativos e 206 como inativos. As características gerais relativas à comparação dos 3 grupos estão descritas na Tabela 1. A porcentagem de indivíduos dos sexos masculino e feminino não diferiu entre os ativos, insuficientemente ativos e os inativos. Houve diferença significante em relação à faixa etária, de modo que houve maior prevalência de indivíduos jovens nos inativos, e de ativos e insuficientemente ativos na meia-idade. A renda familiar também diferiu entre os três grupos, sendo que os ativos e os insuficientemente ativos apresentaram maiores rendas.

Os dados relativos à saúde encontram-se na

Tabela 1. Características gerais dos grupos.

\begin{tabular}{|c|c|c|c|c|c|}
\hline & & $\begin{array}{l}\text { Ativos } \\
\%(n)\end{array}$ & $\begin{array}{c}\text { Tnsuficientemente. Ativos } \\
\%(n)\end{array}$ & $\begin{array}{c}\text { Tnativos } \\
\%(n) \\
\end{array}$ & \\
\hline \multirow[t]{2}{*}{ Sexo } & Feminino & 48 (197) & $44(60)$ & 55 (114) & NS \\
\hline & Masculino & $52(209)$ & $56(77)$ & $45(92)$ & \\
\hline Idade & $<20$ & $26(107)$ & $25(35)$ & $41(84)$ & * \\
\hline \multirow[t]{2}{*}{ (anos) } & 20 a 60 & $45(182)$ & $46(63)$ & $40(83)$ & \\
\hline & $>60$ & $29(117)$ & 28 (39) & 19 (39) & \\
\hline Renda & $<1058$ & $38(145)$ & $38(50)$ & $50(98)$ & * \\
\hline \multirow[t]{2}{*}{$(\mathrm{R} \$)$} & 1058 a 2115 & $23(90)$ & $25(33)$ & $24(48)$ & \\
\hline & $>2115$ & $39(152)$ & $37(49)$ & $26(50)$ & \\
\hline
\end{tabular}

NS - não significante; *- P<0,05 
Tabela 2. Em relação à prevalência de problemas cardíacos, não houve diferença significante entre os grupos. Porém, houve diferença significante na porcentagem de pessoas que possuíam sintomas relacionados à problemas cardíacos, sendo que nos inativos esta porcentagem foi maior que nos ativos e insuficientemente ativos. Houve também diferença significante na utilização de medicamentos, mas neste caso, a prevalência foi maior nos ativos do que nos outros grupos. A prevalência de outros problemas de saúde foi semelhante entre os grupos.

A Tabela 3 mostra a comparação da prevalência dos fatores de risco cardiovasculares entre os grupos. Em relação aos fatores de risco controláveis, não foi verificada diferença significante entre os grupos. Por outro lado, quanto aos fatores de risco não-controláveis, o histórico familiar foi mais prevalente nos inativos, enquanto que o fator sexo-idade foi mais prevalente nos ativos e insuficientemente ativos.

A comparação entre os grupos em relação às medidas realizadas estão descritas na Tabela 4. Não houve diferença significante entre os grupos nas medidas relacionadas à obesidade e à pressão arterial.

Considerando-se o conhecimento sobre a saúde cardiovascular (Tabela 5), a porcentagem de indivíduos que não sabia o valor de seu colesterol foi maior nos inativos do que nos insuficientemente ativos e ativos. O mesmo aconteceu com o conhecimento sobre a glicemia.

É interessante ressaltar que um número maior de indivíduos relatou ter recomendação médica para a prática de atividades físicas nos grupos ativos (39\%) e insuficientemente ativos (34\%) do que nos inativos (25\%).

\section{DISCUSSÃO}

O estudo mostrou que $72 \%$ dos freqüentadores de parques públicos de São Paulo praticavam atividade física de lazer regularmente. Por outro lado, Rego et al. ${ }^{12}$ observaram que apenas $31 \%$ da população adulta de São Paulo realizava atividades físicas de lazer. Além disso, $54 \%$ dos freqüentadores analisados foram classificados como ativos e $18 \%$ como insuficientemente ativos; enquanto que Monteiro et al. ${ }^{19}$ observaram que apenas $3,3 \%$ dos brasileiros eram ativos, e apenas $13 \%$ da população fazia no mínimo 30 minutos de atividade física de lazer pelo menos uma vez por semana. Como o parque é um local propício para a prática de atividade física, a escolha deste local para a pesquisa pode explicar a maior prevalência de pessoas ativas neste que nos demais estudos. De fato, num estudo

Tabela 2. Prevalência de doenças e sintomas cardiovasculares nos grupos.

\begin{tabular}{lcccc}
\hline & Ativos & Insuficientemente. Ativos & Inativos \\
$\%(\mathrm{n})$ & $\%(\mathrm{n})$ & $10(20)$ & $\mathrm{NS}$ \\
\hline Cardiopatia & $13(53)$ & $15(21)$ & $34(70)$ & $*$ \\
Sintomas & $20(80)$ & $20(28)$ & $27(55)$ & NS \\
Outros Problemas & $30(123)$ & $33(45)$ & & 27 \\
\hline
\end{tabular}

NS - não significante; *- P<0,05

Tabela 3. Prevalência de fatores de risco cardiovasculares nos grupos.

\begin{tabular}{lcccc}
\hline & Ativos & Insuficientemente. Ativos & Inativos \\
$\%(\mathrm{n})$ & \\
\hline Colesterol Alto & $\%(\mathrm{n})$ & $19(\mathrm{n})$ & $26(30)$ & $\mathrm{NS}$ \\
Diabetes & $25(75)$ & $7(9)$ & $6(13)$ & $\mathrm{NS}$ \\
Fumo & $8(34)$ & $13(18)$ & $16(32)$ & $\mathrm{NS}$ \\
Hereditariedade & $10(42)$ & $28(38)$ & $35(71)$ & $*$ \\
Hipertensão & $25(100)$ & $22(30)$ & $24(49)$ & $\mathrm{NS}$ \\
Obesidade & $28(112)$ & $12(17)$ & $16(33)$ & $\mathrm{NS}$ \\
Sexo/ldade & $13(54)$ & $45(62)$ & $34(73)$ & $*$ \\
\hline
\end{tabular}

NS - não significante; *- $\mathrm{P}<0,05$

Tabela 4. Prevalência de valores baixos, limítrofes e altos do índice de massa corporal (IMC), índice cintura quadril (ICQ) e pressão arterial (PA) nos grupos.

\begin{tabular}{|c|c|c|c|c|c|}
\hline & & $\begin{array}{l}\text { Ativos } \\
\%(n)\end{array}$ & $\begin{array}{l}\text { Insuficientemente. Ativos } \\
\%(\mathrm{n})\end{array}$ & $\begin{array}{c}\text { Tnativos } \\
\%(n)\end{array}$ & \\
\hline \multirow[t]{3}{*}{ IMC } & $<25$ & $45(182)$ & $48(65)$ & $47(95)$ & NS \\
\hline & 25 a 30 & 42 (168) & $40(54)$ & $37(76)$ & \\
\hline & $\geq 30$ & $13(54)$ & $12(17)$ & $16(33)$ & \\
\hline \multirow[t]{3}{*}{ ICQ } & baixo & 33 (122) & 39 (53) & $33(67)$ & NS \\
\hline & Médio & 35 (143) & $36(49)$ & $38(78)$ & \\
\hline & Alto & 32 (129) & $25(34)$ & $29(59)$ & \\
\hline \multirow[t]{2}{*}{ PA } & $<140 / 90$ & 66 (189) & $72(68)$ & 68 (119) & NS \\
\hline & $\geq 140 / 90$ & $34(96)$ & $28(27)$ & $32(56)$ & \\
\hline
\end{tabular}

NS - não significante; ${ }^{*}$ P $<0,05$ 
Tabela 5. Conhecimento dos valores de glicemia e colesterol nos grupos.

\begin{tabular}{|c|c|c|c|c|c|}
\hline \multirow[t]{2}{*}{ Colesterol } & Sabe & $\begin{array}{c}\text { Ativos } \\
\%(n) \\
74(301)\end{array}$ & $\begin{array}{c}\text { Tnsuficientemente. Ativos } \\
\%(n) \\
69(95)\end{array}$ & $\begin{array}{l}\text { Tnativos } \\
\% \text { (n) } \\
56(115)\end{array}$ & * \\
\hline & Não sabe & $26(105)$ & $31(42)$ & 44 (91) & \\
\hline \multirow[t]{2}{*}{ Glicemia } & Sabe & 72 (294) & $65(89)$ & 54 (111) & * \\
\hline & Não sabe & 28 (112) & $35(48)$ & $46(95)$ & \\
\hline
\end{tabular}

NS - não significante; *- $P<0,05$

anterior, observamos em um parque de São Paulo, que $90 \%$ dos freqüentadores faziam atividades físicas de lazer ${ }^{20}$. Além disso, tem sido evidenciado que $30 \%$ dos indivíduos praticantes de atividades físicas o fazem nos parques $^{21}$. Nos Estados Unidos, 35\% da população que caminha na região urbana, o faz nos parques ${ }^{4}$.

Em relação às características gerais, o presente estudo não observou diferença na porcentagem de indivíduos dos sexos masculino e feminino entre os 3 grupos, mas observou maior prevalência de inativos nos indivíduos mais jovens. Esses resultados são diferentes dos observados por Monteiro et al. ${ }^{19}$ na população brasileira em geral, na qual o grupo dos homens foi mais ativo até os 45-50 anos, e o aumento da idade se associou a uma redução da atividade física de lazer. Novamente, a escolha do parque pode ser responsável por essas diferenças, uma vez que Monteiro et al..$^{19}$ verificaram que com o aumento da idade havia uma preferência por atividades mais moderadas como a caminhada, que normalmente pode ser praticada no parque. Da mesma forma, as mulheres também preferem mais as atividades mais moderadas como a caminhada ${ }^{19}$, o que explica a maior presença delas nos parques igualando-se aos homens.

Em relação à renda, os resultados encontrados corroboram os verificados na literatura ${ }^{19,22}$, ou seja, a prática de atividade física de lazer é mais prevalente nas classes sociais mais altas. É interessante observar, no entanto, que quando são avaliadas também as atividades físicas da vida diária, os resultados são diferentes. Hallal et al. ${ }^{13}$ observaram maior prevalência do sedentarismo na classe socioeconômica mais elevada.

Em relação ao risco cardiovascular, a prevalência dos fatores de risco não diferiu entre os grupos, sendo que os indivíduos inativos apresentaram maior prevalência do fator hereditariedade. De fato, alguns estudos têm sugerido que a propensão genética para a prática de atividade física está associada à propensão genética para as doenças e fatores de risco cardiovascular $^{22}$, de modo que essa pode ser uma explicação para o achado. Porém, os hábitos de vida familiares levam ao sedentarismo e também às doenças cardiovasculares, o que não pode ser descartado. A maior prevalência de indivíduos com o fator sexo/idade presente entre os ativos, como dito anteriormente, pode ser explicada pelo local escolhido para a comparação, ou seja, o parque público facilita a prática de modalidades que normalmente são mais executadas por pessoas do sexo feminino e com idade mais alta ${ }^{13}$.

É importante observar que apesar dos benefícios comprovados da prática regular de atividade física para a prevenção e reabilitação cardiovascular ${ }^{2,5,7}$, a prevalência das doenças cardiovasculares e de seus fatores de risco não diferiu entre os indivíduos ativos, insuficientemente ativos e inativos. Estes resultados sugerem que, talvez, os indivíduos portadores de cardiopatias e fatores de risco cardiovasculares devam ser mais estimulados para a prática de atividade física. Neste sentido, o presente estudo observou a importância da recomendação médica para esta prática, visto que nos grupos ativos e insuficientemente ativos esta recomendação foi mais freqüente do que nos inativos. De fato, a importância da recomendação médica para a adesão à prática de atividade física já foi observada por outros autores ${ }^{21}$.

É importante ressaltar, no entanto, que a prevalência de indivíduos portadores de doenças cardiovasculares foi de $13 \%$ no grupo ativo e $15 \%$ nos insuficientemente ativos, o que demonstra que uma certa parcela dos indivíduos que se exercitam nos parques públicos apresenta um risco cardiovascular alto para esta prática, e fazem parte de uma população cujo teste ergométrico e a supervisão médica inicial são necessários ${ }^{(5)}$. Além disso, o dado mais alarmante é o fato de $20 \%$ dos indivíduos ativos e insuficientemente ativos apresentarem sintomas cardiovasculares, o que os coloca numa classificação de altíssimo risco durante a atividade física, de modo que a prática não é recomendada até uma avaliação mais profunda dos sintomas. Somando-se a isso, observa-se ainda que $51 \%$ dos indivíduos ativos e $45 \%$ dos insuficientemente ativos apresentaram pelo menos um fator de risco cardiovascular e, portanto, possuem um risco moderado para a prática de atividade física. Analisados em conjunto, estes dados demonstram a presença de uma razoável parcela de praticantes de atividades físicas em locais públicos, portadora de um risco cardiovascular moderado a altíssimo, o que enfatiza a necessidade de triagem deste risco e orientação/ prescrição de atividade física nestes locais.

Um outro aspecto interessante levantado pelos dados obtidos é o fato dos indivíduos ativos e insuficientemente ativos apresentarem maior prevalência de conhecimento sobre seu colesterol e glicemia, o que sugere que os indivíduos ativos têm maior conhecimento sobre sua saúde, reforçando a associação entre prática de atividade física e saúde geral.

Obviamente as limitações do presente estudo abrangem todas àquelas relacionadas a estudos observacionais. Além disso, a investigação se limitou a parques públicos da zona oeste de São Paulo e, portanto, os dados estão sujeitos aos vieses da 
população que reside nesta área, porém este aspecto pode ser minimizado pelo fato dos parques incluídos representarem uma grande variedade de classes sociais, o que pode ser observado pela grande variação da renda mensal familiar relatada. Uma outra limitação diz respeito ao fato do estudo ter avaliado apenas as atividades físicas realizadas no período de lazer. Hallal et al. $^{13}$ avaliaram, além da atividade física de lazer, as atividades físicas da vida diária, e observaram resultados distintos deste.

\section{CONCLUSÃO}

Desta forma, os resultados permitem concluir que nos indivíduos freqüentadores de parques públicos de São Paulo, os ativos e insuficientemente ativos são mais velhos e tem maior renda que os inativos. Além disso, os ativos e insuficientemente ativos apresentam a mesma prevalência de doenças e de fatores de risco cardiovasculares modificáveis dos inativos, mas têm menos sintomas e maior conhecimento sobre sua saúde. Somando-se a isso, estes indivíduos apresentam recomendação médica para a prática de atividade física com maior freqüência. Estes resultados indicam que grande parte dos praticantes de atividade física em locais públicos apresenta risco cardiovascular de moderado a altíssimo para a prática de atividades físicas, o que ressalta a importância da triagem de risco cardiovascular e posterior orientação/prescrição para esta prática nos locais públicos.

\section{REFERÊNCIAS BIBLIOGRÁFICAS}

1. Benseñor I, Lotufo P. Estado atual do tratamento e controle do diabetes melito, da dislipidemia e da hipertensão arterial no Brasil e no mundo - Condutas gerais para a adequada manutenção do controle. In: Mion Jr. D, Nobre F, editores. Risco Cardiovascular Global III. São Paulo: Lemos Editorial; 2002. p. 17-43.

2. Blair SN, Cheng Y, Holder JS. Is physical activity or physical fitness more important in defining health benefits? Med Sci Sports Exerc 2001;33(6 Suppl):S37999; discussion S419-20.

3. Kohl HW. Physical activity and cardiovascular disease: evidence for a dose response. Med Sci Sports Exerc 2001;33(6 Suppl):S472-83.

4. Eyler AA, Brownson RC, Bacak SJ, Housemann RA. The epidemiology of walking for physical activity in the United States. Med Sci Sports Exerc 2003;35(9):152936.

5. American College of Sports Medicine. ACSM's Guidelines for Exercise Testing and Prescription. Philadelphia: Lippincott - Williams and Wilkins; 2006.

6. Sociedade Brasileira de Cardiologia, Sociedade Brasileira de Hipertensão, Sociedade Brasileira de Nefrologia. V Diretrizes Brasileiras de Hipertensão Arterial. Arq Bras Cardiologia 2007;89(3):e24-e79.

7. Forjaz CL, Negrão CE. Sedentarismo. In: Mion JR D, Nobre F, editores. Risco Cardiovascular Global. São Paulo: Lemos Editorial; 1999. p. 139-162.
8. Cornelissen VA, Fagard RH. Effects of endurance training on blood pressure, blood pressure-regulating mechanisms, and cardiovascular risk factors. Hypertension 2005;46(4):667-75.

9. Pescatello LS, Franklin BA, Fagard R, Farquhar WB, Kelley GA, Ray CA. American College of Sports Medicine position stand. Exercise and hypertension. Med Sci Sports Exerc 2004;36(3):533-53.

10. Boule NG, Haddad E, Kenny GP, Wells GA, Sigal RJ. Effects of exercise on glycemic control and body mass in type 2 diabetes mellitus: a meta-analysis of controlled clinical trials. JAMA 2001;286(10):1218-27.

11. Leon AS, Sanchez OA. Response of blood lipids to exercise training alone or combined with dietary intervention. Med Sci Sports Exerc 2001;33(6 Suppl): S502-15.

12. Rego RA, Bernardo FA, Rodrigues SS, Oliveira ZM, Oliveira MB, Vasconcellos C, et al. Risk factors for chronic non-communicable diseases: a domiciliary survey in the municipality of Sao Paulo, SP (Brazil). Methodology and preliminary results. Rev Saude Publica 1990;24(4):277-85.

13. Hallal PC, Matsudo SM, Matsudo VK, Araujo TL, Andrade DR, Bertoldi AD. Physical activity in adults from two Brazilian areas: similarities and differences. Cad Saude Publica 2005;21(2):573-80.

14. Matsudo SM, Matsudo VR, Araujo TL, Andrade DR, Andrade EL, de Oliveira LC, et al. The Agita Sao Paulo Program as a model for using physical activity to promote health. Rev Panam Salud Publica 2003;14(4):265-72.

15. Mittleman MA, Maclure M, Tofler GH, Sherwood JB, Goldberg RJ, Muller JE. Triggering of acute myocardial infarction by heavy physical exertion. Protection against triggering by regular exertion. Determinants of Myocardial Infarction Onset Study Investigators. N Engl J Med 1993;329(23):1677-83.

16. American College of Sports Medicine Position Stand and American Heart Association. Recommendations for cardiovascular screening, staffing, and emergency policies at health/fitness facilities. Med Sci Sports Exerc 1998;30(6):1009-18.

17. Mion Jr. D, Silva, H. Device to correct the reading of blood pressure according to the patient's arm circumference. J Hypertension 1986; 4(suppl.15):S581.

18. Craig CL, Marshall AL, Sjostrom M, Bauman AE, Booth $\mathrm{ML}$, Ainsworth BE, et al. International physical activity questionnaire: 12-country reliability and validity. Med Sci Sports Exerc 2003;35(8):1381-95.

19. Monteiro CA, Conde WL, Matsudo SM, Matsudo VR, Bonsenor IM, Lotufo PA. A descriptive epidemiology of leisure-time physical activity in Brazil, 1996-1997. Rev Panam Salud Publica 2003;14(4):246-54.

20. Forjaz CL, Tinucci T, Bartholomeu T, Fernandes TE, Casagrande V, Massucato JG. Assessment of the cardiovascular risk and physical activity of individuals exercising at a public park in the city of sao paulo. Arq Bras Cardiol 2002;79(1):35-50.

21. King AC, Blair SN, Bild DE, Dishman RK, Dubbert PM, Marcus $\mathrm{BH}$, et al. Determinants of physical activity and interventions in adults. Med Sci Sports Exerc 1992;24(6 Suppl):S221-36.

22. Rankinen T, Bray MS, Hagberg JM, Perusse L, Roth SM, Wolfarth $B$, et al. The human gene map for performance and health-related fitness phenotypes: the 2005 update. Med Sci Sports Exerc 2006;38(11):1863-88.

\section{Endereço para correspondência}

Cláudia Lúcia de Moraes Forjaz

Av. Prof. Mello Moraes, 65 - Butantã -

CEP 05508-900 São Paulo - SP - Brasil

E-mail: cforjaz@usp.br
Recebido em 20/07/07

Revisado em 17/10/07

Aprovado em 18/01/08 